\title{
The correlation between sarcopaenia and post-transjugular intrahepatic portosystemic shunt hepatic encephalopathy: a single-institution review
}

\author{
Zahava C. Farkas, Tariq Rashid, Ying Sharon Chen, Tausif Mohamed Siddiqui, Srikanth Yandrapalli, \\ Shalom Frager, Wilbert S. Aronow, Roxana Bodin, Shekher Maddineni
}

Department of Medicine, Division of Vascular Interventional Radiology and Division of Gastroenterology and Hepatobiliary Disease, Westchester Medical Centre and New York Medical College, Valhalla, USA

Submitted: 25 April 2019

Accepted: 26 April 2019

Arch Med Sci Atheroscler Dis 2019; 4: e89-e93

DOI: https://doi.org/10.5114/amsad.2019.85380

Copyright (c) 2019 Termedia \& Banach

\section{Abstract}

Introduction: Sarcopaenia, or muscle wasting, can be used to objectively quantify malnutrition in cirrhotic patients.

Material and methods: In this retrospective study, a list of all patients who underwent transjugular intrahepatic portosystemic shunt (TIPS) procedure at Westchester Medical Centre from September 2009 to July 2018 was obtained, and individual chart reviews were performed.

Results: In total, 90 charts were reviewed. Fifty-six patients satisfied our inclusion criteria. Using PMA cut-off values determined in prior studies, we found that 50 of the 56 patients in our study were sarcopaenic. The majority of the patients were male $(n=45)$. The most common aetiology of cirrhosis was alcoholic cirrhosis $(n=27)$, followed by viral hepatitis $(n=10)$, and the most common indication for TIPS was refractory ascites $(n=34)$. The mean age in the sarcopaenic group was 60.1 years compared to 57.4 years in the non-sarcopaenic group. Mean MELD-Na scores and albumin levels were comparable in both groups. Only one patient was deceased at 6 months postTIPS. Of the 56 patients included, 18 developed clinically significant hepatic encephalopathy within 6 months of their TIPS procedure. All 18 patients belonged to the sarcopaenic group; 6 patients were not sarcopaenic, and none of them were noted to develop HE within 6 months of their TIPS $(p=0.074)$ Conclusions: Based on our results, we concluded that sarcopaenia correlates with the development of hepatic encephalopathy within 6 months of a TIPS procedure; however, the results did not reach statistical significance.

Key words: sarcopaenia, cirrhosis, transjugular intrahepatic portosystemic shunt.

\section{Introduction}

Transjugular intrahepatic portosystemic shunt (TIPS) is a common therapeutic procedure for cirrhotic patients with complications secondary to portal hypertension, including refractory ascites, hydrothorax, or variceal bleeding. For many patients with end-stage liver disease, TIPS is a bridge to liver transplantation. The most frequent complication of the TIPS procedure is hepatic encephalopathy $(\mathrm{HE})$, with an incidence of $25 \%$ to $45 \%$ [1]. The HE is of great clinical importance because it affects pa-

\author{
Corresponding author: \\ Wilbert S. Aronow MD, FACC, \\ FAHA \\ Cardiology Division \\ Westchester Medical \\ Centre and New York \\ Medical College \\ Macy Pavilion \\ Room 141, Valhalla \\ NY 10595, USA \\ Phone: (914) 493-5311 \\ Fax: (914) 235-6274 \\ E-mail: wsaronow@aol.com
}


tient prognosis, quality of life, time to liver transplant and is associated with increased mortality [2]. Therefore, identifying factors that predispose a patient to post-TIPS HE will allow for appropriate patient selection and improved outcomes.

The conventional metrics for peri-procedural risk stratification in cirrhotic patients are the model for end-stage liver disease (MELD), the newer MELD-Na score, and the Child-Turcotte-Pugh (CTP) score. Research has shown that a higher MELD score, hyponatraemia, and higher total bilirubin level are associated with the development of overt $\mathrm{HE}$ post-TIPS [3]. Additionally, the incidence of $\mathrm{HE}$ is higher in patients with increased age, pre-existing $\mathrm{HE}$, and higher CTP score $[4,5]$. However, neither the MELD nor the CTP scoring systems take into account factors such as nutritional status or functional disability.

Malnutrition is the most common complication of cirrhosis, and recent studies have suggested that a strong link exists between poor nutritional status and poor outcomes in patients with cirrhosis. Compared to serum albumin and body mass index (BMI), both of which may be modified by hepatopathy or ascites, sarcopaenia, or muscle wasting, offers a more objective measurement of nutritional status and holds promise as a predictor of patient outcome after TIPS [6].

The purpose of our study was to investigate this relationship and the utility of incorporating such data into pre-procedure risk calculators. Given the clinical implications, it is of great benefit to the interprofessional medical team to have a better understanding of the clinical factors that can affect patients with end-stage liver disease undergoing the TIPS procedure.

\section{Material and methods}

This study was approved by New York Medical College Institutional Review Board (IRB). A list of all patients who underwent a TIPS procedure at Westchester Medical Centre (WMC) from September 2009 to July 2018 was obtained, and individual chart reviews were performed. A total of 90 cases were evaluated for inclusion in the study. For inclusion, patients were required to: have a documented diagnosis of cirrhosis, have undergone TIPS for the first time at WMC during the above-mentioned time period, have serologic data available within 1 week prior to the date of the TIPS procedure, have imaging from computed tomography (CT) or magnetic resonance imaging (MRI) performed within 1 month of the TIPS, and have 6-month follow-up data.

In total, 56 cases met our inclusion criteria. From the original cohort, seven patients did not have adequate serologic data, 2 patients were excluded because their TIPS was unsuccessful, $10 \mathrm{pa}$ - tients did not have the required imaging studies available in our electronic medical record system, and 15 patients were excluded due to lack of 6-month follow-up data.

Detailed chart reviews were performed by two of the primary investigators. Prior to performing statistical analysis, all data were reviewed simultaneously by both investigators to ensure that consistent methods were used to collect each piece of information. Relevant clinical information including age, gender, BMI, aetiology of cirrhosis, indication for TIPS, albumin levels, serology needed to calculate MELD-Na, the presence of HE both pre- and post-TIPS, and 6-month mortality rates were collected and analysed for the purposes of this study. The presence or absence of HE pre- and post-TIPS was based on detailed review of the patient's chart, looking for specific mention of the condition or treatment of it (i.e. the use of lactulose or rifaximin).

To date, there is no clinically established criterion for sarcopaenia. However, there is a general consensus that it can be measured using a combination of functional, biological, and imaging-related markers. Multiple modalities have been proposed as ideal ways to measure muscle mass, each with varying limitations. Dual-energy X-ray absorptiometry (DEXA), for example, is widely available, inexpensive and exposes the patient to minimal radiation; however, it may overestimate muscle mass due to its inability to differentiate water from bone-free lean tissue. The CT and MRI are considered the "gold standard" techniques because they allow simultaneous evaluation of fat and muscle [7, 8].

For the purposes of this study, CT and MRI were utilised to calculate psoas muscle area (PMA) using the Synapse PACS system at the L2-L3 levels as an indicator of muscle mass. The PMA has been shown to offer better accuracy than alternative metrics, including L3 skeletal muscle index (L3S$\mathrm{MI})$, in defining sarcopaenia in cirrhotic patients [6]. Images from CTs or MRIs of the abdomen taken within one month of the TIPS procedure were used to calculate the PMA (sum of the area of the two psoas muscles at the level of the third or fourth lumbar vertebra) for each patient. Based on data published by Golse et al. in 2017, sarcopaenia was defined as a PMA less than $1561 \mathrm{~mm}^{2}$ for men and less than $1464 \mathrm{~mm}^{2}$ for women.

\section{Statistical analysis}

All statistical analyses were performed using SPSS Statistical Software v25.0 (IBM, Chicago, IIlinois). A bivariate analysis with Pearson $\chi^{2}$ test was used to compare the demographics and outcome of interests between the study groups. We utilised the $t$-test to compare the differences of 
continuous variables between the study groups. Two-sided $p$-values were reported, and values below 0.05 were considered statistically significant.

\section{Results}

\section{Demographics and clinical characteristics}

Statistical analysis was performed on a final cohort of 56 patients. The mean age was 58 years, with a male predominance of $80.4 \%$. The most common aetiology of cirrhosis was alcoholic cirrhosis $(n=27)$, followed by viral hepatitis $(n=10)$, primary biliary sclerosis $(n=5)$, cryptogenic cirrhosis $(n=4)$, non-alcoholic steatohepatitis (NASH) $(n=2)$, autoimmune hepatitis $(n=1)$, and those with a combination of the above-mentioned aetiologies $(n=7)$. The most common indication for TIPS was refractory ascites $(n=34)$, followed by variceal bleeding $(n=15)$ and recurrent hydrotho$\operatorname{rax}(n=4)$.

\section{Predictors of post-TIPS hepatic encephalopathy}

Fifty (89.2\%) patients were sarcopaenic. In our patient population, the overall rate of development of HE after TIPS procedure was noted to be 32 per 100 patients $(n=18)$. In the sarcopaenic group, 36 per 100 patients $(n=18)$ developed HE within 6 months of their TIPS procedure, in comparison to no patients in the non-sarcopaenic group.

The mean age of the patients who developed HE (mean: 60.1) was higher than in patients who did not develop HE (mean: 57.4) $(p=0.349)$.

The mean MELD-Na score for those who developed HE within 6 months post-TIPS was 15.6, compared to a mean of 15.1 in the group who did not develop HE within 6 months of the TIPS ( $p=$ 0.731).

Pre-TIPS albumin levels were lower in patients with sarcopaenia (mean: 2.9), as compared to those who were not sarcopaenic (mean: 3.1). There appeared to be linear correlation between serum albumin levels and PMA (correlation coefficient $r=0.244, p=0.069$ ); however, the difference was not statistically significant. Among the patients who were sarcopaenic, the mean albumin in the group that developed HE was also lower at 2.8 (compared to 3.0) in those who did not develop HE $(p=0.058)$.

\section{All-cause mortality}

We were unable to analyse the association of sarcopaenia with post-TIPS mortality due to the very low number of deaths reported in our study cohort. While there was no mortality in the non-sarcopaenic group, there was 1 patient with sarcopaenia who died within 6 months of their TIPS.

\section{Discussion}

Sarcopaenia, or age-related loss of skeletal muscle mass, is thought to play a key role in the outcome of chronic diseases. The aetiology of sarcopaenia is incompletely understood but is believed to be multifactorial and characterised by a variety of molecular changes in muscle tissue [9]. Although the effects of muscle wasting have been extensively studied in the setting of acute illness and linked to poor outcomes, limited data are available on the impact that sarcopaenia has on the progression of chronic disease, such as liver cirrhosis [10]. The aim of our study was to systematically review the impact of sarcopaenia on post-TIPS HE, a common complication seen in the cirrhotic population.

Portal hypertension is a common complication of cirrhosis, defined as an increase in the blood pressure of the portal venous system greater than $4 \mathrm{~mm} \mathrm{Hg}$ above the hepatic vein pressure or greater than $6 \mathrm{~mm} \mathrm{Hg}$ above the right atrial pressure [11]. The TIPS is an effective and widely used method of treating complications of portal hypertension such as recurrent ascites and variceal bleeding; however, its use has been limited by two serious complications: shunt stenosis and hepatic encephalopathy.

The prevalence of overt $\mathrm{HE}$ at the time of diagnosis of cirrhosis is $10-14 \%$ in general, $16-21 \%$ in those with decompensated cirrhosis, and $10-50 \%$ in patients with transjugular intrahepatic portosystemic shunt [12-14]. The HE is one of the most debilitating manifestations of liver disease, greatly impacting both patients and their caregivers. It is associated with a high risk of recurrence, reduced quality of life and poor survival [2]. Furthermore, it results in utilisation of more health care resources in adults than other manifestations of liver disease; according to one study, patients with HE accounted for approximately 110,000 hospitalisations yearly in the United States from 2005-2009 [15]. Therefore, examining risk factors that may increase the likelihood of its occurrence is important.

The Model for End-Stage Liver Disease (MELD) and Child-Turcotte-Pugh (CTP) scoring systems are used to assess the severity of chronic liver disease and prognosticate outcomes in patients with cirrhosis. The major limitation of the MELD and CTP scores is that they do not take into account the patient's nutritional or functional status. Incorporating sarcopaenia into such scoring systems may bridge this gap. Montano-Loza et al. found that in a cohort of 669 patients being evaluated for liver transplant "modification of MELD to include sar- 
copaenia is associated with improved prediction of mortality in patients with cirrhosis, primarily in patients with low MELD scores" [16]. Across the board, however, results remain inconclusive [17]. Many more questions remain to be asked on this topic; this study specifically aimed to answer if sarcopaenia was a risk factor for worse outcomes after TIPS, as measured by the development of HE.

Although there is no consensus on the exact definition of sarcopaenia, there is a general agreement that it includes both a functional parameter such as muscle performance (i.e. grip strength and gait speed) and a quantitative index such as muscle mass. Myostatin inhibits cell growth, and increased serum concentrations in mice have been reported to cause muscle atrophy [9]. Myostatin and major myostatin-interacting proteins are therefore hypothesised to play a key role in the development of human sarcopaenia. However, several reports have failed to demonstrate this correlation, and therefore we cannot use such markers to diagnose sarcopaenia [18]. In this retrospective study, we were unable to collect data on muscle performance or strength, and so, based on recently published data by Golse et al., we analysed the prognostic value of skeletal muscle mass alone, using the psoas muscle area [6].

In this study, the majority of patients who underwent TIPS at our institution met criteria for sarcopaenia. A correlation was seen between sarcopaenia and the development of post-TIP $\mathrm{HE}$; however, our results did not reach statistical significance, possibly due to the small patient sample. We were unable to analyse the correlation between sarcopaenia and 6-month all-cause mortality post-TIPS due to the very low number of reported deaths - there was just one mortality within the cohort.

There are multiple limitations to consider during future research endeavours on this topic. Obtaining adequate imaging and serologic data is difficult using retrospective analysis. We found that patients often had imaging and bloodwork performed at outside institutions and lacked available data within our medical records system. In addition, these tests were often performed outside of our designated time frames. Manually calculating each patient's PMA from CT or MRI, conducted by one radiologist for consistency, requires a significant amount of time.

The presence or absence of HE post-TIPS was based on the review of inpatient hospital records. This meant that if a patient was treated for mild $\mathrm{HE}$ by an outpatient physician, there would be no record of it in our system. Additionally, our institution is a liver transplant centre and major referral centre; therefore, patients often come from long distances for liver transplant evaluation or proce- dures such as TIPS and then follow-up closer to home for routine care or hospitalisations. For this reason, many patients lacked 6-month follow-up data in our system and had to be excluded from the original cohort.

In conclusion, modification of the MELD score to include sarcopaenia may lead to improved prediction of poor outcomes, including mortality in patients with cirrhosis. Consistent with recently published data, we found that the presence of sarcopaenia correlated with poorer outcomes - in our case, a higher incidence of HE within a 6-month period after TIPS; however, our results did not reach statistical significance. We postulate that in conjunction with the MELD score, sarcopaenia should be taken into consideration during a preTIPS evaluation, and its presence should alert clinicians to the potential impact it will have on patient outcomes. Optimising a patient's nutritional status, using aggressive physical therapy and nutritional supplementation, should be a possible goal before proceeding with TIPS. Additional prospective studies are needed to solidify these theories and lead to its incorporation into illness severity and peri-procedural risk calculators in the cirrhotic population. Furthermore, identifying a serologic marker of sarcopaenia would greatly contribute to this field of study and should be a priority. We also found that multiple limitations exist in studying the effects of sarcopaenia on patient outcomes, and these limitations should be considered when conducting future research on this topic.

Limitations: 1) This is a retrospective study. 2) There is no conclusive definition for sarcopaenia or agreement on how to measure it. Most groups agree that the definition of sarcopaenia simultaneously includes a functional parameter (i.e. muscle performance) and a quantitative index (i.e. muscle mass). We were only able to incorporate a quantitative metric in this study, using psoas muscle area. 3) Close to $90 \%$ of the patients in this study were sarcopaenic. This left only a small control group of patients who were non-sarcopaenic. 4) Imaging used to measure PMA all took place within one month of the TIPS but at varying periods. Significant decline in muscle mass can occur in a short period of time, especially in those who are critically ill. Therefore, a patient who was deemed non-sarcopaenic may have progressed to meet criteria for sarcopaenia between the time imaging was performed and when the TIPS procedure takes place. 5) The presence of HE was based on chart reviews from hospital admissions with an admission diagnosis or problem list that included $\mathrm{HE}$ or mention of treatment of the condition (i.e. lactulose or rifaximin). Patients may have developed $\mathrm{HE}$ without requiring hospitalisation or 
were hospitalised outside of our institution. Due to lack of access to outpatient records or hospital records outside of our institution, the incidence of HE may be over- or underestimated.

Despite these limitations, our study has the following important strengths: 1) Detailed chart reviews were performed for each patient included in the cohort. 2) Metrics used in other pre-op risk calculators such as MELD-Na are continuous variables with day-to-day variation. Incorporating sarcopaenia, an objective measure of malnutrition, may have important implications and requires further research.

\section{Conflict of interest}

The authors declare no conflict of interest.

\section{References}

1. He F, Dai S, Xiao Z, et al. Pathological predictors of shunt stenosis and hepatic encephalopathy after transjugular intrahepatic portosystemic shunt. Biomed Res Int 2016; 2016: 3681731.

2. Vilstrup H, Amodio P, Bajaj J, et al. Hepatic encephalopathy in chronic liver disease: 2014 practice guideline by the American Association for the Study of Liver Diseases and the European Association for the Study of the Liver. Hepatology 2014; 60: 715-35.

3. Merola J, Chaudhary N, Qian M, et al. Hyponatremia: a risk factor for early overt encephalopathy after transjugular intrahepatic portosystemic shunt creation. J Clin Med 2014; 3: 359-72.

4. Peter P, Andrej Z, Katarina SP, et al. Hepatic encephalopathy after transjugular intrahepatic portosystemic shunt in patients with recurrent variceal hemorrhage. Gastroenterol Res Pract 2013; 2013: 398172.

5. Bai M, Qi X, Yang Z, et al. Predictors of hepatic encephalopathy after transjugular intrahepatic portosystemic shunt in cirrhotic patients: a systematic review. J Gastroenterol Hepatol 2011; 26: 943-51.

6. Golse N, Bucur PO, Ciacio O, et al. A new definition of sarcopenia in patients with cirrhosis undergoing liver transplantation. Liver Transpl 2017; 23: 143-54.

7. Cesari M, Fielding RA, Pahor M, Vellas B; International Working Group on Sarcopenia, et al. Biomarkers of sarcopenia in clinical trials-recommendations from the International Working Group on Sarcopenia. J Cachexia Sarcopenia Muscle 2012; 3: 181-90.

8. Abellan van Kan G, Houles M, Vellas B. Identifying sarcopenia. Curr Opin Clin Nutr Metab Care 2012; 15: 436-41.

9. Ratkevicius A, Joyson A, Selmer I, et al. serum concentrations of myostatin and myostatin-interacting protiens do not differ between young and sarcopenic elderly men. J Gerontol A Biol Sci Med Sci 2011; 66: 620-6.

10. Puthucheary ZA, Rawal J, McPhail M, et al. Acute skeletal muscle wasting in critical illness. JAMA 2013; 310: 1591-600.

11. Pinzani M, Rosselli M, Zuckermann M. Liver cirrhosis. Best Pract Res Clin Gastroenterol 2011; 25: 281-90.

12. Saunders JB, Walters JR, Davies AP, Paton A. A 20-year prospective study of cirrhosis. Br Med J 1981; 282: 263-6.

13. Nolte W, Wiltfang J, Schindler C, et al. Portosystemic hepatic encephalopathy after transjugular intrahepatic portosystemic shunt in patients with cirrhosis: clinical, laboratory, psychometric, and electroencephalographic investigations. Hepatology 1998; 28: 1215-25.

14. Papatheodoridis GV, Goulis J, Leandro G, et al. Transjugular intrahepatic portosystemic shunt compared with endoscopic treatment for prevention of variceal rebleeding: a meta-analysis. Hepatology 1999; 30: 61222.

15. Stepanova M, Mishra A, Venkatesan C, Younossi ZM. In-hospital mortality and economic burden associated with hepatic encephalopathy in the United States from 2005 to 2009. Clin Gastroenterol Hepatol 2012; 10: 1034-41.

16. Montano-Loza AJ, Duarte-Rojo A, Meza-Junco J, et al. Inclusion of sarcopenia within MELD (MELD-Sarcopenia) and the prediction of mortality in patients with cirrhosis. Clin Transl Gastroenterol 2015; 6: e102.

17. Kim G, Kang SH, Kim MY, Baik SK. Prognostic value of sarcopenia in patients with liver cirrhosis: a systematic review and meta-analysis. PLoS One 2017; 12: e0186990.

18. White TA, LeBrasseur NK. Myostatin and sarcopenia: opportunities and challenges - a mini-review. Gerontology 2014; 60: 289-93. 